\title{
Электрополевое поведение резонансных особенностей в туннельной составляющей фототока в гетероструктурах InAs(QD)/GaAs
}

\author{
(С) М.Л. Орлов ${ }^{1,3}$, Н.С. Волкова ${ }^{2}$, Н.Л. Ивина ${ }^{1}$, Л.К. Орлов ${ }^{3,4}$ \\ ${ }^{1}$ Нижегородский институт управления РАНХиГС, \\ 603950 Нижний Новгород, Россия \\ ${ }^{2}$ Нижегородский государственный университет им. Н.И. Лобачевского, \\ 603950 Нижний Новгород, Россия \\ ${ }^{3}$ Институт фризики микроструктур Российской академии наук, \\ 603950 Нижний Новгород, Россия \\ ${ }^{4}$ Нижегородский государственный технический университет им. Р.Е. Алексеева, \\ 603950 Нижний Новгород, Россия \\ E-mail: orlov@ipm.sci-nnov.ru
}

(Получена 10 июля 2017 г. Принята к печати 24 июля 2017 г.)

\begin{abstract}
Изучено электрополевое поведение резонансных особенностей, наблюдаемых на фотоэлектрических характеристиках гетерокомпозиций InAs/GaAs. Обсуждается механизм эмиссии возбуждаемых светом носителей заряда из квантовых точек InAs в матрицу GaAs. Показано, что при температуре жидкого азота величина фототока в сильном поперечном электрическом поле определяется исключительно эффектом туннелирования электронов сквозь барьер, формируемый в окрестности интерфейсов квантовых точек. Сопоставление экспериментальных кривых с квазиклассическим выражением для туннельной составляющей тока и последующий анализ структуры потенциала позволили уточнить значения параметров исследуемой гетерокомпозиции. Проанализирован вклад в общую величину туннельного тока резонансной составляющей, связанной с возможным туннелированием электронов сквозь барьер с участием локальных уровней дефектов на гетерогранице. Проведен теоретический анализ влияния уровня возбуждения системы на величину фототока, протекающего через гетеропереход InAs/GaAs.
\end{abstract}

DOI: 10.21883/FTP.2018.09.46148.8683

\section{1. Введение}

Проблема выращивания и использования на практике массивов квантовых точек на базе самых разнообразных материалов широко обсуждается в литературе уже более трех десятилетий. Повышенное внимание к гетерокомпозициям на основе соединений $\mathrm{A}^{\mathrm{III}} \mathrm{B}^{\mathrm{V}}$ связано как с работами по лазерам на квантовых точках [1-3], так и с возможностью формирования в перспективе на базе соответствующих многослойных структур одномерных квантовых сверхрешеток [2,3], интерес к которым не ослабевает, несмотря на значительные трудности, которые приходится преодолевать на пути их реализации. В настоящее время проблема получения гетероструктур, содержащих в матрице GaAs двумерный массив квантовых точек InAs, решается с использованием как методов низкотемпературной молекулярно-лучевой эпитаксии (MBE) [4,5], так и методом МОС-гидридной эпитаксии (газофазной эпитаксии из металлорганических соединений) [6-8]. Однако для получения нужных характеристик формируемых последним способом нанообъектов в условиях относительно высоких по сравнению с МВЕ температур и скоростей роста приходится использовать ряд специальных приемов [6]. В работе [8] для получения на поверхности GaAs однородного массива квантовых точек InAs применялось предварительное покрытие поверхности GaAs монослоем атомов висмута. Висмут в данной системе наряду с адсорбируемым водородом выступает в качестве сурфактанта, ограничивающего ми- грационную подвижность атомов In, As на поверхности растущего слоя и препятствующего как росту крупных кластеров с дислокациями, так и коалесценции мелких островков.

В наноструктурированных квантовых гетерокомпозициях области интерфейсов занимают объем, сравнимый с объемом низкоразмерных включений, оказывая в ряде случаев определяющее влияние на их свойства. С морфологическими особенностями границ, уровнем их дефектности и неоднородностью легирования прилегающих областей связаны не только характеристики рассеивающего пограничного потенциала [9], но и возможность проявления в окрестности гетерограниц дополнительных состояний [10]. Особенно актуальна эта проблема для сильно напряженных гетерокомпозиций, в которых границы слоев вследствие формирования интенсивных деформационных полей являются геттерами разнообразных точечных дефектов. Особенности потенциала, в том числе связанные с присутствием в окрестности интерфейсов заряженной примеси, могут заметным образом сказаться на электрических характеристиках системы при протекании тока как вдоль, так и поперек плоскости слоев структуры.

Наибольшее внимание структуре гетерограниц уделяется в плоскослоистых канальных системах, широко используемых в устройствах микроэлектроники. Замечено, что присутствие локализованных состояний на интерфейсах транспортного канала оказывает негативное влияние на характеристики устройств, особенно в 
условиях разогрева электронного газа. Примером такого рода явлений может служить, в частности, наличие падающего участка на выходных характеристиках полевого транзистора в тянущем электрическом поле, большем порогового поля $E_{\mathrm{th}}$. При этом одновременно нарастает ток в поперечном плоскости слоев направлении вследствие либо диффузионного [11,12], либо туннельного [13-15] переноса электронов между соседними слоями структуры. В обоих случаях наблюдается также заметное снижение величины порогового поля $E_{\text {th }} \mathrm{c}$ уменьшением длины транспортного канала, что связывают со снижением амплитуды потенциального барьера на интерфейсах вследствие возрастания поперечной составляющей тока за счет туннелирования электронов через состояния дефектов на гетерогранице.

\section{2. Структура и спектральные характеристики образцов}

Далее особенности эффекта туннелирования электронов сквозь барьер на гетерогранице и возможное проявление в туннельном токе локальных поверхностных состояний изучаются с использованием метода фотоэлектронной спектроскопии на примере структуpы InAs/GaAs. Гетерокомпозиции выращивались методом МОС-гидридной эпитаксии и содержали вблизи поверхности структуры однородный массив квантовых точек (КT) InAs с размерами в плоскости структуры $D_{\mathrm{QD}} \approx 60$ нм и высотой островка $h_{\mathrm{QD}} \approx 8$ нм [8]. Рассматриваемая система характеризуется предельно высоким (> 20\%) несоответствием постоянных кристаллических решеток контактирующих материалов. Возникающие поля упругих напряжений обусловливают высокую вероятность появления в окрестности гетерограниц структуры локальных состояний, связанных как с дефектами решетки кристалла, так и с геттерируемой примесью. Особую роль при этом может играть высаживаемый на поверхность GaAs монослой атомов $\mathrm{Bi}$, который, являясь изовалентной примесью, способен изменить природу поверхностных состояний за счет замещения висмутом атомов $\mathrm{Ga}(\mathrm{In})$ в решетке GaAs (InAs).

Для рассматриваемой системы $\mathrm{GaAs} / \mathrm{InAs}(\mathrm{QD}) /$ GaAs [8] можно предположить, что отдельные островки InAs в структуре подвержены со стороны GaAs главным образом всестороннему сжатию, определяемому только компонентой тензора деформаций $C_{11}=8.5 \cdot 10^{5}$ кг $/ \mathrm{cm}^{2}$. Данное предположение основано на том, что толщина островков InAs в псевдоморфных структурах InAs/GaAs значительно превосходит толщину смачивающего слоя, а толщина покрывающего слоя GaAs во много раз превосходит вертикальный размер островков InAs. Оценка ширины запрещенной зоны $E_{g}$ в деформированных KT InAs c учетом того, что для InAs барический коэффициент ширины запрещенной зоны составляет $\left(d E_{g} / d P\right)_{T} \approx 3.2 \cdot 10^{-6}$ эВ/кг см $^{2}$, дает величину $E_{g}(\mathrm{InAs}) \approx 0.95$ эВ. Эта величина согласуется с результатами, полученными методом емкостной спектроскопии [16]. Для ширины запрещенной зоны $\mathrm{GaAs}$ далее принималось значение $E_{g}(\mathrm{GaAs}) \approx 1.5$ эB, а для глубины InAs квантовых ям в зоне проводимости, в соответствии с общепринятыми представлениями, значение $U_{\mathrm{QD}} \approx 0.3$ эВ.

Ширина барьера на уровне основного состояния квантовой точки $E_{1}$ для системы с концентрацией доноров в буферном слое $N_{D} \approx 3 \cdot 10^{16} \mathrm{~cm}^{-3}$ и максимально резкими границами определяется выражением $L_{1}=$ $=W\left(U_{\mathrm{QW}}-E_{1}\right) /\left(\psi_{0}-U\right)$, где $W=\left\{2 \varepsilon_{S}\left(\psi_{0}-U\right) / e N_{D}\right\}^{1 / 2}-$ ширина области пространственного заряда, $U_{\mathrm{QW}}-$ глубина квантовой ямы, $\psi_{0} \approx 0.8 \mathrm{~B}-$ значение поверхностного потенциала для $\mathrm{GaAs}, \varepsilon_{S}=\varepsilon_{r} \varepsilon_{0}=$ $=1.16 \cdot 10^{-12} \Phi / \mathrm{cm}$ - диэлектрическая проницаемость GaAs. Оценка ширины барьера при малой величине прикладываемого внешнего напряжения $(U \approx 0)$ дает значение $L_{1} \approx 50$ нм, что обусловливает чрезвычайно низкую величину туннельной составляющей тока. Структура потенциала квантовой ямы и барьера, отделяющего квантовую точку InAs от континуума $\mathrm{GaAs}$, однако, сильно зависит как от уровня легирования буферного подслоя GaAs, так и от степени и характера расплывания образующегося в окрестности интерфейсов состава тройного соединения $\operatorname{In}_{1-x} \mathrm{Ga}_{x} \mathrm{As}$. Более точный расчет методом конечных разностей формы потенциала с учетом возможного размытия гетерограниц $\Delta x_{1}=\Delta x_{2} \approx 2$ нм при $\quad \psi_{0}-U \approx 1$ эВ дает значение ширины барьера на высоте первого уровня размерного квантования в яме $L_{1} \approx 25$ нм. При расчетах для толщин слоев, в соответствии с $[8,17]$, для нижнего буферного слоя $\mathrm{GaAs}$ : Ві выбиралось значение $d_{1}=0.6$ мкм, для InAs - значение $d_{2}=h_{\mathrm{QD}}=8 \mathrm{HM}$, для верхнего покрывающего слоя GaAs $-d_{3}=20 \mathrm{Hм}$. $\mathrm{C}$ ростом напряжения на контактах до значения $U \approx 3.0$ В ширина $L_{1}$ приближается к значению 2.5 нм и туннельная составляющая тока становится определяющей в общем токе системы.

Для анализа электронных состояний в структурах, содержащих в окрестности приповерхностного потенциала ансамбли квантовых точек, традиционно применяется метод фотоэлектронной спектроскопии $[4,5,17,18]$. Детальный анализ данным методом температурных зависимостей фотопроводимости показал, что в диапазоне температур $T>100 \mathrm{~K}$ в фототоке, наблюдаемом в указанных выше структурах, доминирует исключительно термоактивационная составляющая, характер поведения которой определяется особенностями термического выброса электронов из квантовых ям.

В настоящей работе с целью исключения влияния термоэмиссионных эффектов обсуждаются только результаты измерений, выполненные при температуре жидкого азота. Основное внимание обращено на электрополевые зависимости спектральных характеристик изучаемых гетерокомпозиций и выявление механизма туннельной эмиссии возбуждаемых светом электронов из квантовых точек InAs в матрицу GaAs через барьер, формируемый 


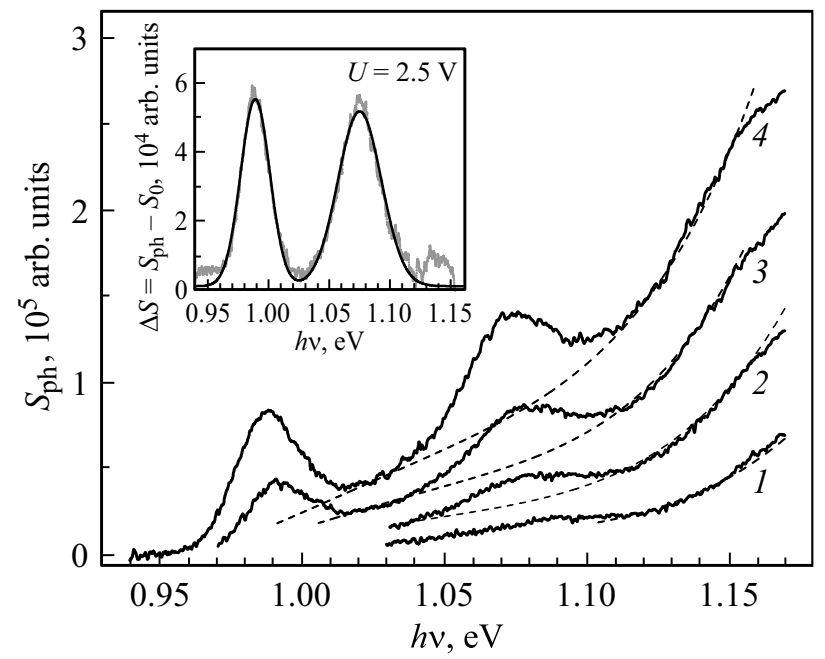

Рис. 1. Спектры фоточувствительности $S_{\mathrm{ph}}$ структуры $\mathrm{GaAs} / \mathrm{InAs} / \mathrm{GaAs}$ с квантовыми точками InAs при температуpe $80 \mathrm{~K}$ для прикладываемого к барьеру Шоттки напряжения $U=1$ (1), 1.5 (2), 2 (3), 3 В (4). Штриховые линии базовая нерезонансная составляющая фотопроводимости $S_{0}$. На вставке - резонансные пики $\Delta S=S_{\mathrm{ph}}-S_{0}$, полученные для $S_{\mathrm{ph}}$, соответствующей $U=2.5 \mathrm{~B}$ (точки); сплошные линии - аппроксимации экспериментальных пиков гауссовыми кривыми.

на гетерогранице. Обсуждается возможное участие в туннельном токе пограничных состояний и уровней дефектов в окрестности барьера. Типичные спектры фоточувствительности $S_{\mathrm{ph}}$, получаемые на барьере Шоттки для структуры с одиночным слоем квантовых точек, показаны на рис. 1. Измерения проводились при температуре жидкого азота для серии значений прикладываемого к структуре внешнего электрического поля. Пики фоточувствительности $S_{\mathrm{ph}}$ в спектрах структуры при энергии фотонов $h v \approx 0.99$ эВ $(h v \approx 1.075$ эВ) связаны с оптическими переходами в квантовой точке между дискретными уровнями основного состояния дырки и основного (возбужденного) состояния электрона $E_{1}\left(E_{2}\right)$.

\section{3. Низкотемпературная полевая зависимость фототока}

Рассмотрим влияние сильного электрического поля на поведение наблюдаемых резонансных пиков в измеряемом фототоке. Цель проводимого исследования заключалась в том, чтобы понять, насколько хорошо экспериментальные зависимости соответствуют теоретическим кривым простейшего вида $[16,19]$, описывающим туннелирование электронов сквозь потенциальный барьер треугольной формы. Используемые для анализа резонансные пики $\Delta S_{i}$ (см. вставку на рис. 1) были выделены нами из наблюдаемых спектров путем вычитания из экспериментальных зависимостей $S_{\text {ph }}$ (рис. 1 , сплошные линии) базовых, аппроксимируемых полиномом 4-й степени кривых $S_{0}$ (рис. 1, штриховые линии). Вид выделенных в чистом виде резонансных пиков с $i=1,2$ для одного частного случая, соответствующего $U=2.5 \mathrm{~B}$, показан на вставке к рис. 1. Каждый из выделенных пиков с высокой степенью точности описываются функцией Гаусса: $\Delta S_{i}=A_{0 i}+A_{1 i} \exp \left[-2\left(E-E_{i}^{\max }\right)^{2} / w_{i}^{2}\right]$. Здесь $w_{i}=(\mathrm{FWHM})_{i} /(\ln 4)^{1 / 2}$, где FWHM - ширина пика на полувысоте, определяемая разбросом размеров КТ относительно их среднего значения. Размытие резонансных пиков (FWHM) в диапазоне рассматриваемых полей составляет величину $\sim 25$ мэВ для первого пика $(i=1)$ и 35-45 мэВ для второго пика $(i=2)$. Изменение с ростом поля положения уровней, определяемое по сдвигу положения максимумов спектральных линий $E_{i}^{\max }$, составляет величину $\sim 10$ мэВ.

На рис. 2 представлены зависимости от величины прикладываемого поля $F$ площадей $\left(Q_{i}\right)$ под соответствующими резонансными кривыми (символы - кружки, полученные путем анализа гауссианов, аппроксимирующих спектральные пики). Поле $F$ связано с введенной ранее величиной прикладываемого к структуpe напряжения $U$ соотношением $F / F_{0}=\left(1+U / \psi_{0}\right)^{1 / 2}$. Ограничиваемые спектральными кривыми площади $\left(Q_{i}\right)$ линейным образом связаны с амплитудой соответствующих резонансных пиков $\left(\Delta S_{i}\right): Q_{i}=(\pi / 2)^{1 / 2} \Delta S_{i} w_{i}$. Анализ зависимости площадей под резонансными пиками, а не амплитуд пиков, от величины прикладываемого поля позволяет в косвенной форме учесть вклад в ошибку измерений разброс квантовых точек по размерам.

Проведем сопоставление результатов фотоэлектрических измерений с расчетами, выполненными на базе простейшей, широко используемой в литературе квазиклассической формулы

$$
\begin{aligned}
\Xi_{i}(F) & =\left[e F / 4\left(2 m_{e} \Delta_{i}\right)^{0.5}\right] \exp \left[-4 \Delta_{i}\left(2 m_{e} \Delta_{i}\right)^{0.5} / 3 e \hbar F\right] \\
& =a_{i} F \exp \left(-b_{i} / F\right),
\end{aligned}
$$

описывающей вероятность туннелирования электронов с уровня $E_{i}$ потенциальной ямы сквозь барьер треугольной формы (эффект Франца-Келдыша) [16,19]. Учет только электронной составляющей фототока основан на малости в условиях пониженных температур эффектов туннелирования, связанных с токами, обусловленными тяжелыми дырками. Подзона легких дырок вследствие размерного квантования сдвигается по энергии вниз, поэтому легкие дырки в туннельном переносе не принимают участие. Заметим также, что выражение для туннельного тока с учетом дырок из обеих подзон сильно усложняется вследствие перепутывания под барьером состояний в подзонах легких и тяжелых дырок [20], что заставляет пренебрегать дырочной составляющей и в термоактивационном токе [17], наблюдаемого в условиях эксперимента, проводимого при повышенных температурах. В формуле (1) $m_{e}$ - масса электронов в $\mathrm{GaAs}, \Delta_{i}$ - высота барьера, разделяющего состояния электрона на $i$-м уровне квантовой точки и континуума 


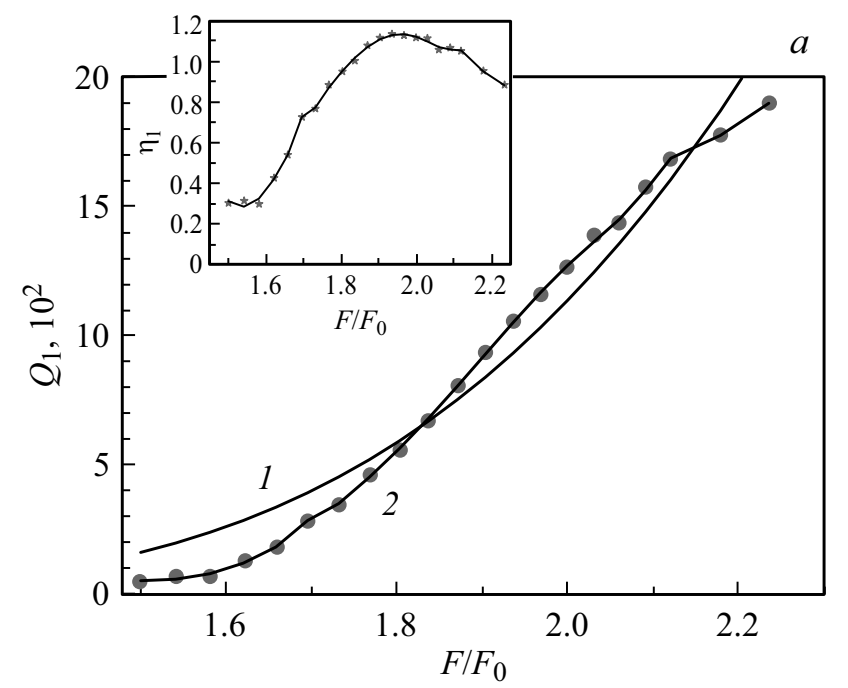

$b$

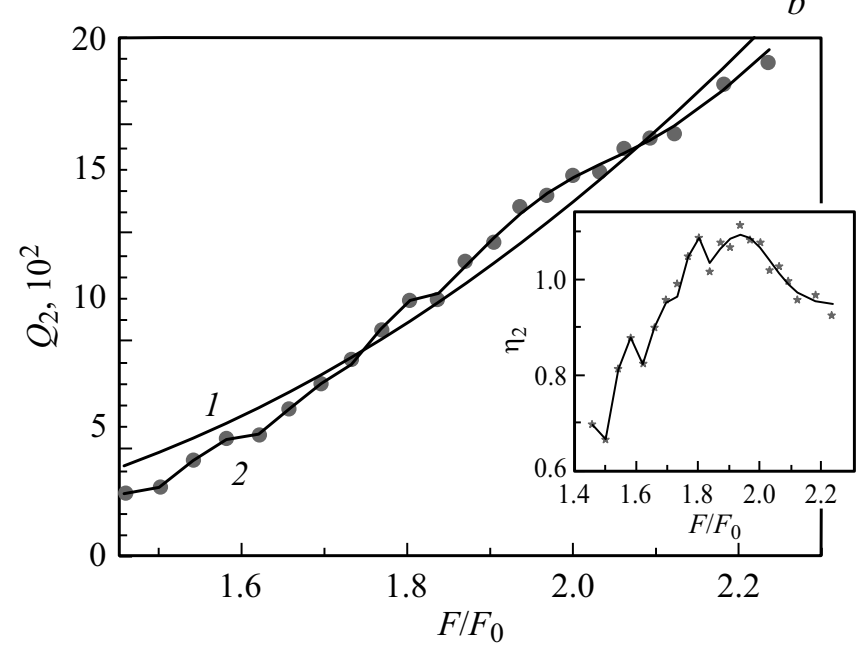

Рис. 2. Зависимости величины фототока (точки), определяемого площадями под кривыми первого $(a) h v_{1}=0.99$ эВ и второго $(b), h v_{2}=1.075$ эВ, резонансов (см. вставку на рис. 1 ), от величины прикладываемого поля $F / F_{0} .1$ - аппроксимация $\Xi_{i}(F)$ согласно $(1)$ при $a_{1}\left(a_{2}\right)=0.864 \cdot 10^{5}\left(1.348 \cdot 10^{4}\right)$, $b_{1}\left(b_{2}\right)=10.047(5.596), \eta_{i}=1 ; 2-$ с учетом зависимости $\eta_{i}(F)$. На вставках - зависимости $\eta_{i}(F)=\Delta Q_{i} / \Xi_{i}(F)$ (точки), сплошные линии - аппроксимация суммой гауссовых кривых.

в слое GaAs. На рис. 2 кривые 1 получены с использованием формулы (1), кривые 2 - с использованием дополнительного подгоночного множителя $\eta_{i}(F)$ (см. вставки к рис. 2, $a, b)$. Кривые 1 на рис. $2, a, b$ являются результатом подгонки по методу сопряженных градиентов теоретической зависимости (1) к экспериментальным данным (точки). Здесь для напряженности поля в барьере Шоттки принято $F(U, x)=\left(e N_{0} / \varepsilon_{r} \varepsilon_{0}\right)(W-x)$, где $W$ - ширина области пространственного заряда, $N_{D}$ - уровень легирования донорами структуры, $\psi_{0}-$ высота барьера на границе, $U$ - напряжение, прикладываемое к барьеру Шоттки. Далее при вычислениях будем полагать $F=F_{0}\left(1+U / \psi_{0}\right)^{1 / 2}$, а высоту барьера $\psi_{0}$ на контакте Шоттки к GaAs положим равной $0.8 \mathrm{~B}$. В этом случае наилучший результат подгонки достигается для значений параметров: $a_{1}\left(a_{2}\right)=0.864 \cdot 10^{5}\left(1.348 \cdot 10^{4}\right)$, $b_{1}\left(b_{2}\right)=10.047(5.596)$.

Представленные на рис. 2 зависимости свидетельствуют о доминирующей роли туннельной составляющей в протекающем по структуре токе, одновременно показывая заметное несовпадение теоретических кривых 1 и экспериментальных данных 2 (точки) на рис. 2. Одной из причин наблюдаемого несоответствия данных может являться, в частности, влияние прикладываемого электрического поля на форму потенциала квантовой точки, приводящее к сдвигу положения уровней размерного квантования. Другая причина может быть связана с пренебрежением дырочной составляющей в туннельном токе. Проведенные расчеты показывают, однако, что в рассматриваемом диапазоне полей изменение положения уровней не превышает 10\% от глубины ямы, а величина туннельной составляющей тока тяжелых дырок пренебрежимо мала. Вследствие этого влияние указанных факторов на вид обсуждаемых кривых вполне можно не учитывать.

Сопоставление экспериментальных данных с квазиклассическим выражением (1) для туннельной составляющей тока позволяет параллельно уточнить некоторые значения параметров структуры. Знание на основании данных фотоэлектронной спектроскопии (см. рис. 1) разности положений уровней энергий в яме $\Delta_{1}-\Delta_{2}=E_{2}-E_{1}=\left[3 e \hbar F_{0} / 4\left(2 m_{e}\right)^{0.5}\right]^{2 / 3}$ $\times\left(b_{1}^{2 / 3}-b_{2}^{2 / 3}\right) \approx 0.085$ эВ позволяет оценить величину поля $F_{0}=4\left(2 m_{e}\right)^{0.5}\left[\left(E_{2}-E_{1}\right) /\left(b_{1}^{2 / 3}-b_{2}^{2 / 3}\right)\right]^{3 / 2} / 3 e \hbar$. Полагая $\psi_{0}=0.8 \mathrm{~B}$ и зная значения соответствующих параметров $b_{1}$ и $b_{2}$ для поля $F_{0}$, получим $F_{0} \approx 2.24 \cdot 10^{5} \mathrm{~B} / \mathrm{cm}$. Знание параметра $F_{0}$ позволяет в свою очередь на основании формулы (1) провести оценку эффективной высоты барьера $\Delta_{1}=\left[3 e \hbar F_{0} b_{1} / 4\left(2 m_{e}\right)^{0.5}\right]^{2 / 3}$ относительно основного состояния электронов в квантовой яме: $\Delta_{1} \approx 280$ мэВ. Учитывая возможную погрешность параметра $\psi_{0}$ в диапазоне значений $\psi_{0}=(0.8 \pm 0.1) \mathrm{B}$ нетрудно определить допустимый интервал значений искомых величин. Для $F_{0}$ соответственно имеем $\delta F_{0} \approx 0.2 \cdot 10^{5} \mathrm{~B} / \mathrm{cm}$, для $\Delta_{1}$ разброс значений составляет величину $\delta \Delta_{1} \approx 15$ мэВ. Таким образом, согласно проведенным оценкам, высота барьера при туннелировании электронов с первого уровня КТ составляет величину $\Delta_{1} \approx(280 \pm 10)$ мэВ, глубина потенциальной ямы для электронов примерно равна 300-350 мэВ. Критерием применимости соотношения (1), согласно [16], является выполнение неравенства $e \hbar F_{0} / 2\left(2 m_{e}\right)^{1 / 2}>k T \Delta_{1}^{1 / 2}$. $\mathrm{B}$ рассматриваемой нами системе в условиях азотных температур имеем $2.4 \cdot 10^{-30}>0.9 \cdot 10^{-30}$ Дж ${ }^{3 / 2}$, т.е. при полях $F>F_{0}$ туннельный ток явно доминирует над термоэмиссионным током.

Наиболее естественной причиной наблюдаемого на рис. 2 отклонения теоретической зависимости (кривые 1) от полевых зависимостей, получаемых в эксперименте (точки), является присутствие в области потенциального барьера положительно заряженных локальных 
центров. Последние могут быть связаны как с дефектами кристаллической решетки, так и с геттерируемыми к границе из нижележащего слоя GaAs атомами примеси. Наличие заряженных пограничных состояний в барьере может приводить к появлению дополнительной неоднородной составляющей в плотности туннельного тока, объясняющей отклонение теоретической зависимости, полученной для идеального треугольного барьера (рис. 2, кривая 1), от данных, наблюдаемых в эксперименте (рис. 2, точки). Влияние пограничных состояний в барьере на величину туннельного тока можно учесть, умножая выражение (1) на функцию $\eta_{i}(F)$, зависящую от поля и отражающую характер случайного распределения локальных центров по энергии и ширине потенциального барьера в окрестности гетерограницы. Проанализируем на качественном уровне вид функции $\eta_{i}(F)$. Соответствующие кривые представлены на вставках к рис. 2, $a, b$. Полученные зависимости описываются суммой гауссианов, что указывает на присутствие в области GaAs потенциального барьера локальных центров с разной энергией залегания и расположенных на разном расстоянии от границы. Появление таких центров вблизи нижней границы KT InAs, в частности, можно связать и с присутствием в области гетероперехода монослоя атомов Bi, выступающего, с одной стороны, в качестве сурфактанта, а с другой - в качестве изовалентной примеси по отношению к GaAs и InAs. $\mathrm{C}$ ростом поля положение локальных уровней в барьере смещается по энергии относительно положения уровней размерного квантования в KT InAs. Пересечение с ростом поля уровней локальных центров под барьером и уровней квантовых точек будет приводить к появлению дополнительной составляющей в туннельном токе, а в идеальном варианте и к возможности проявления на полевой зависимости слабо выраженных резонансных всплесков тока, аналогично тому, как это наблюдалось для системы $\mathrm{Si} / \mathrm{Si}_{1-x} \mathrm{Ge}_{x}$ в работе [15].

Вероятное участие поверхностных состояний в туннельном токе подтверждается и видом спектральных кривых, представленных на рис. 1. На кривых, кроме резонансных пиков, наблюдается (штриховые линии) компонента фототока $S_{0}$, спадающая с уменьшением энергии фотонов практически по экспоненте и связанная с хвостом плотности состояний в запрещенной зоне GaAs. Явно выраженная зависимость интенсивности фототока $S_{0}(h v)$ от поля $F$ говорит об участии подбарьерных состояний в окрестности гетерограницы в захвате возбуждаемых электронов с последующим туннельным перебросом их в континуум состояний нижележащего слоя GaAs. Для заданной частоты света $v_{i}$ в поле $F$ нетрудно определить интегральную эффективность $A S_{0}$ поглощения света пограничными состояниями, проводя интегрирование базовой функции $S_{0}$ в диапазоне частот от минимального значения $v_{0} \quad\left(h v_{0}=0.94\right.$ эВ $)$ до значения, равного частоте $v_{i}$. Серия найденных зависимостей $A S_{0}(F)$ показана на рис. 3, a. Точки $1-9$ при заданном значении поля соответствуют площадям
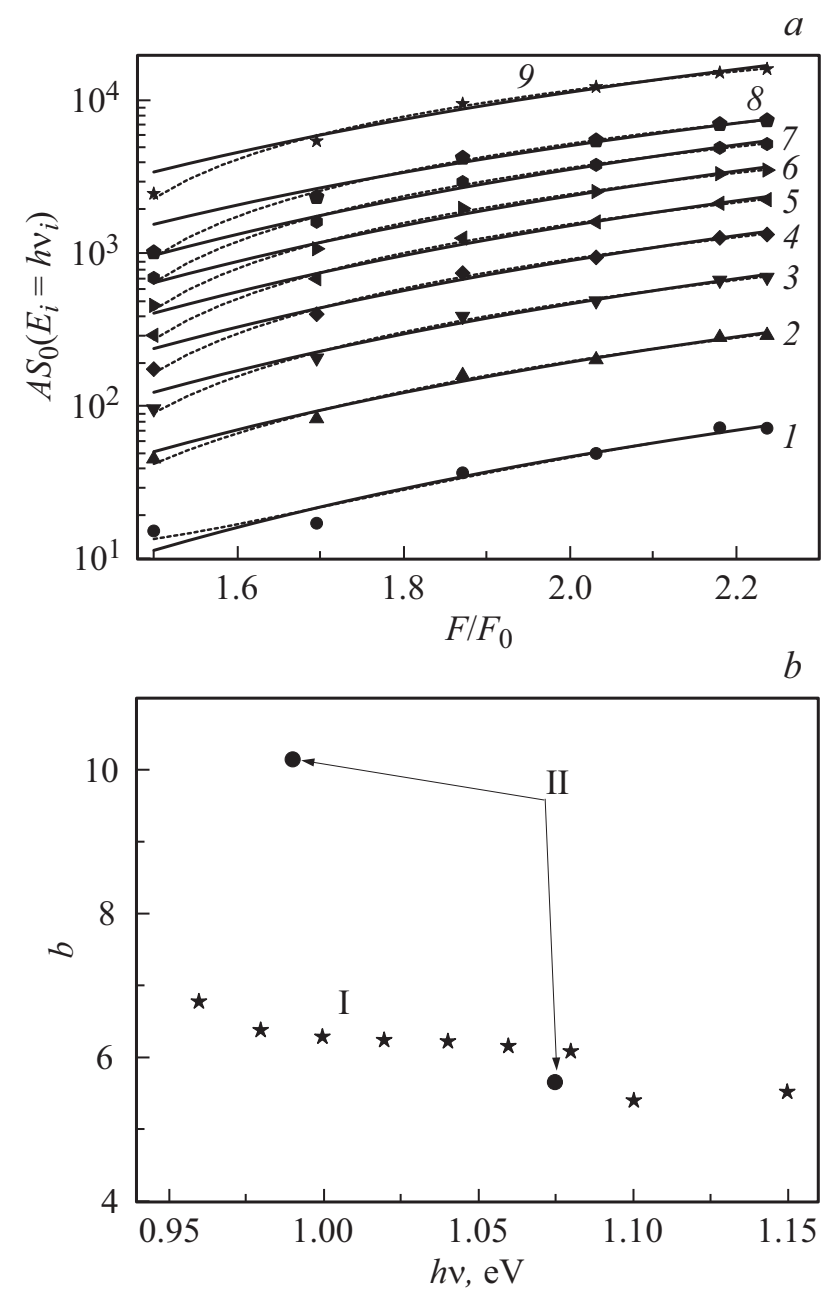

Рис. 3. Интегральная площадь (символы) под базовой спектральной кривой $S_{0}(a$, точки) в зависимости от прикладываемого поля для энергий фотонов $h v=0.96(1), 0.98(2), 1.0$ (3), 1.02 (4), 1.04 (5), 1.06 (6), 1.08 (7), 1.1 (8), 1.15 (9); штриховые линии - параболическая аппроксимация, сплошные линии аппроксимация соотношением (1) со значениями параметра $b$, представленного на рис. $3, b$ точками I; точки II соответствуют значениям параметров $b_{1}, b_{2}$, найденным для резонансных частот $v_{1}, v_{2}$ на рис. 2 .

$A S_{0}$ под спектральной кривой $S_{0}$ с верхним значением частоты $v_{i}$, соответствующим энергиям фотонов $h v_{i}=0.96,0.98,0.1,1.02,1.04,1.06,1.08,1.1,1.15$ эВ. На рис. 3, $a$ пунктирными линиями показана параболическая аппроксимация вычисленных значений площадей $A S_{0}$, сплошными линиями - их аппроксимация соотношением (1). Найденные значения параметра $b$, используемого в формуле (1), в зависимости от частоты излучения приведены на рис. $3, b$ (точки I). Точками II здесь показаны значения параметров $b_{1}, b_{2}$, найденные для резонансных частот $v_{1}, v_{2}$ ранее (см. рис. 2). Принимая значение $F_{0} \approx 2.24 \cdot 10^{5} \mathrm{~B} / \mathrm{cm}$, для характерной высоты барьера по отношению к среднему уровню энергии, соответствующему максимальной плотности состояний дефектов на границе, получаем $\Delta_{0} \approx 200$ мэВ. Данная 
величина соответствует высоте барьера на уровне возбужденного состояния квантовой ямы.

\section{4. Влияние уровня засветки на характеристики барьера и величину фототока}

Выше были рассмотрены фотоэлектрические характеристики диодной структуры со слоем квантовых точек в условиях низкого уровня засветки. Показана возможность использования туннельного эффекта для того, чтобы призошло рассасывание заряда электронов, накапливаемого в слое квантовых точек с целью достижения максимальной скорости переключения сигнала. При рассмотрении фотоэффекта, однако, была полностью проигнорирована возможность накопления дырок в валентной зоне КТ, что может критическим образом сказаться на переключающих характеристиках диодной структуры. В связи с этим представляется важным провести анализ особенностей накопления и характера распределения носителей заряда обоих знаков по слоям структуры, а также влияния накапливаемого заряда на потенциал в системе. В реальных устройствах важна не только скорость рассасывания, но и величина накапливаемого заряда. Высокая плотность заряда в базовой области гетеродиода нанометровой толщины может быть реализована путем засветки структуры импульсами фемтосекундного лазера.

Далее для лучшего понимания процессов, протекающих в гетероструктуре $p$-GaAs/InAs(QD) $/ n$-GaAs, проведен анализ распределения носителей заряда по слоям системы и изучено влияние заряда, накапливаемого в базе гетеродиода, на структуру потенциала и вид вольт-амперных характеристик. Расчет потенциала в направлении, перпендикулярном плоскости слоев рассматриваемой гетероструктуры (ось $x$ ), проведен на базе системы дифференциальных уравнений, включающей в себя уравнения квазигидродинамики и уравнение Пуассона. В стационарном случае рабочая система уравнений записывается в следующем виде:

$$
\begin{gathered}
\operatorname{div}\left(j_{n}\right)=R_{n}-G_{n}, \quad \operatorname{div}\left(j_{p}\right)=R_{p}-G_{p}, \\
j_{n}=e\left[\mu_{n} n E_{x}+D_{n}(d n / d x)\right], \\
j_{p}=e\left[\mu_{p} p E_{x}-D_{p}(d p / d x)\right], \quad j=j_{n}+j_{p}+j_{s}, \\
\left(d \varphi^{2} / d x^{2}\right)=\left(e / \varepsilon_{r} \varepsilon_{0}\right)\left(n+N_{A}-p-N_{D}\right) .
\end{gathered}
$$

Здесь $n, p$ - концентрации электронов и дырок в заданной плоскости структуры; $j_{n}, j_{p}$ - плотности электроного и дырочного токов, $\varphi(x)$ - электростатический потенциал, $N_{D}, N_{A}-$ концентрации доноров и акцепторов в слоях активной части структуры, задаваемые условиями задачи. В рамках используемого диффузионно-дрейфового приближения $\mu_{n}, \mu_{p}-$ подвижности носителей заряда в отдельных слоях; $F_{x}$ - напряженность электрического поля поперек плоскости слоев структуры, $D_{e}$ и $D_{h}-$ коэффициенты диффузии, связанные со значениями $\mu_{e}$ и $\mu_{h}$ соотношениями Эйнштейна. Ток смещения записывается в стандартной форме $j_{s}=e D_{p} p_{0} / L_{p}+e D_{n} n_{0} / L_{n}$, где $p_{0}, n_{0}-$ равновесные концентрации неосновных носителей, $L_{n}$ и $L_{p}$ - диффузионные длины неравновесных электронов и дырок. Темп генерации электронов и дырок в единице объема $(G)$, вызываемый внешними, в частности тепловыми, факторами, и скорость рекомбинации неравновесных носителей $(R)$ связаны с указанными выше величинами соотношениями

$$
\begin{gathered}
G(x, \lambda)=A \alpha(\lambda) I_{0} \exp [-\alpha(\lambda) x], \\
R=\left[p(x) n(x)-n_{i}^{2}\right] /\left[\tau_{n}\left(n+n_{1}\right)+\tau_{p}\left(p+p_{1}\right)\right], \\
n_{1}=n_{i} \exp \left(-E_{t} / k_{\mathrm{B}} T\right), \quad p_{1}=n_{i}^{2} / n_{1},
\end{gathered}
$$

$E_{t}$ - уровень энергии рекомбинационного центра.

Основные уравнения дополнены граничными условиями, которые выбирались из условия электронейтральности и термодинамического равновесия системы на контактах:

$$
\begin{gathered}
p_{0} n_{0}=n_{i}^{2}, \quad p+N_{D}-n-N_{A}=0, \\
\varphi(x=0)=-\ln \left[p(x=0) / n_{i}\right], \\
\varphi(x=L)=-\ln \left[n(x=L) / n_{i}\right] .
\end{gathered}
$$

Время жизни носителей полагалось равным

$$
\begin{gathered}
\tau_{p(n)}=\tau_{p(n)} \text { при } 0<x<x_{s}, \\
\tau_{p(n)} / s \text { при } x>x_{n}-x_{s},
\end{gathered}
$$

где $s$ - отношение времен жизни носителей заряда в объеме слоя GaAs и на его внешней поверхности, $x_{s}$ - толщина поверхностного слоя GaAs с повышенным уровнем рекомбинации неравновесных носителей заряда, $x_{n}$ - координата нижней границы легированного донорами слоя $\mathrm{GaAs}$ в структуре.

Решение системы дифференциальных уравнений (2) находилось численным методом с использованием метода сеток. При расчете формы потенциала учитывалась возможность расплывания основного и примесного состава слоев в окрестности интерфейсов на величину $\Delta_{1} / 2\left(\Delta_{2} / 2\right)$ в обе стороны от границы. Предполагалось, что состав в пограничных слоях на толщинах $\Delta_{1}\left(\Delta_{2}\right)$, а соответственно и ряд других характеристик, напрямую связанных с составом материала, меняется по гармоническому закону. Расчеты, выполненные с использованием представленной выше схемы для ряда значений параметров, приведены на рис. 4. В модели при проведении вычислений выбирались следующие значения параметров: общая толщина структуры (в нм)

$$
D=\delta_{1 s}+d_{1}(\mathrm{I})+\Delta_{1}+d_{2}(\mathrm{II})+\Delta_{2}+d_{3}(\mathrm{III})+\delta_{3 s}
$$

с $d_{1}=20 \mathrm{Hм}, d_{2}=8 \mathrm{HM}, d_{3}=100 \mathrm{Hм}$, значения $\Delta_{1}$ и $\Delta_{2}$ задавались в интервале от 2 до $8 \mathrm{HM}, \delta_{1 s}=\delta_{3 s}=5 \mathrm{HM}-$ области поверхностной рекомбинации, $N_{D}, N_{A}=$ $=10^{16}-10^{18} \mathrm{~cm}^{-3}, E_{g}(\mathrm{GaAs})=1.5$ эB, $E_{g}(\mathrm{InAs})=0.95$ эВ, 

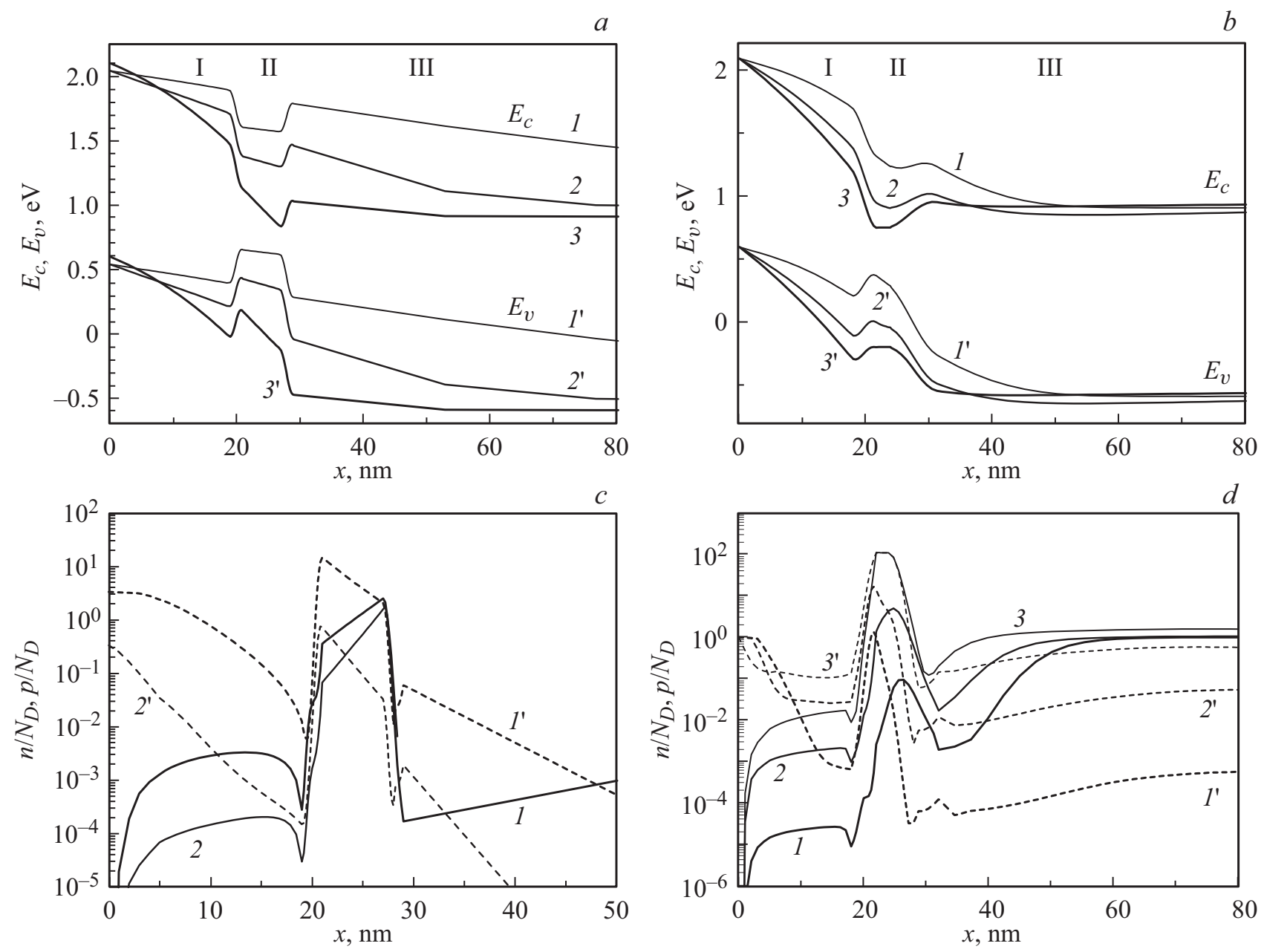

Рис. 4. Энергетические зоны $(a, b)\left(E_{c}-\right.$ зона проводимости, $E_{v}$ - валентная зона) и распределение концентрации неравновесных носителей заряда $(c, d)(n$ - электронов, сплошные линии, $p$ - дырок, штриховые линии) в структуре $\mathrm{GaAs}(\mathrm{I}) / \mathrm{InAs}(\mathrm{II}) / \mathrm{GaAs}(\mathrm{III})$. a, $c: N_{A}=10^{17}\left(1,1^{\prime}, 2,2^{\prime}\right), 10^{16} \mathrm{~cm}^{-3}\left(3,3^{\prime}\right) ; N_{D}=10^{17}, 0.3 \cdot 10^{17}\left(1,1^{\prime}\right), 3 \cdot 10^{14}\left(2,2^{\prime}\right), 10^{16} \mathrm{~cm}^{-3}\left(3,3^{\prime}\right) ; \Delta_{1}=\Delta_{2}=2\left(1^{\prime}, 3,3^{\prime}\right)$, $4 \mathrm{Hм}\left(2,2^{\prime}\right) . b, d: N_{A}=N_{D}=1 \cdot 10^{18} \mathrm{~cm}^{-3} ; \Delta_{1}=4 \mathrm{Hм}, \Delta_{2}=8$ нм. Интенсивность падающего на образец света: $a, c-I_{0}=100$; $b, d-I_{0}=10^{2}\left(1,1^{\prime}\right), 10^{4}\left(2,2^{\prime}\right), 10^{5}\left(3,3^{\prime}\right)$.

разрыв валентной зоны $\Delta E_{v}(\mathrm{GaAs} / \mathrm{InAs})=0.3$ эВ. При расчетах нами рассмотрена ситуация резких границ $\Delta_{1}=\Delta_{2}=2$ нм (рис. $\left.4, a, c\right)$ и неблагоприятная ситуация сильного размытия гетерограниц $\Delta_{1}, \Delta_{2}$ (рис. $4, b, d$ ), достаточно часто реализуемая на практике в условиях использования не прецизионных высокотемпературных методов роста.

Проведенные вычисления характеристик потенциала, формируемого в зоне проводимости в окрестности слоя InAs, и распределения между слоями возбуждаемых светом неравновесных носителей заряда представлены на рис. 4. Как и следовало ожидать, форма потенциального рельефа сильно зависит как от уровня легирования слоев $\mathrm{GaAs}$, так и от степени размытия гетерограниц (рис. 4, $a, b$ ). Асимметрия потенциала в условиях низкого уровня засветки (рис. 4,c) приводит к смещению плотности заряда, связанной с электронами и дырками, к противоположным границам квантовой ямы. Возрастание концентрации неравновесных носите- лей заряда в квантовых точках при повышении уровня засветки структуры (см. рис. 4, $d$ и 5, $a$ ) также приводит к изменению формы потенциала как квантовой ямы, так и барьеров в прилегающих слоях GaAs (рис. 4,b). Нужно отметить, что в данной системе в условиях низкого уровня возбуждения концентрация тяжелых дырок вследствие высокой плотности состояний более, чем на порядок, превышает концентрацию электронов. Поэтому можно ожидать, что при температурах > $200 \mathrm{~K}$ вклад дырок в величину наблюдаемого термоэмиссионного тока может быть вполне заметен, т.е. пренебрегать дырочной компонентой тока при анализе температурных зависимостей фототока, как это делается в большинстве вышеупомянутых работ $[4,5,17]$, вообще говоря, нельзя. При повышении уровня засветки (рис. 5,a) происходит выравнивание концентраций неравновесных электронов и дырок в слое квантовых точек InAs и образование в них сгустков низкотемпературной квазинейтральной электронно-дырочной плазмы. Плотность электронно- 

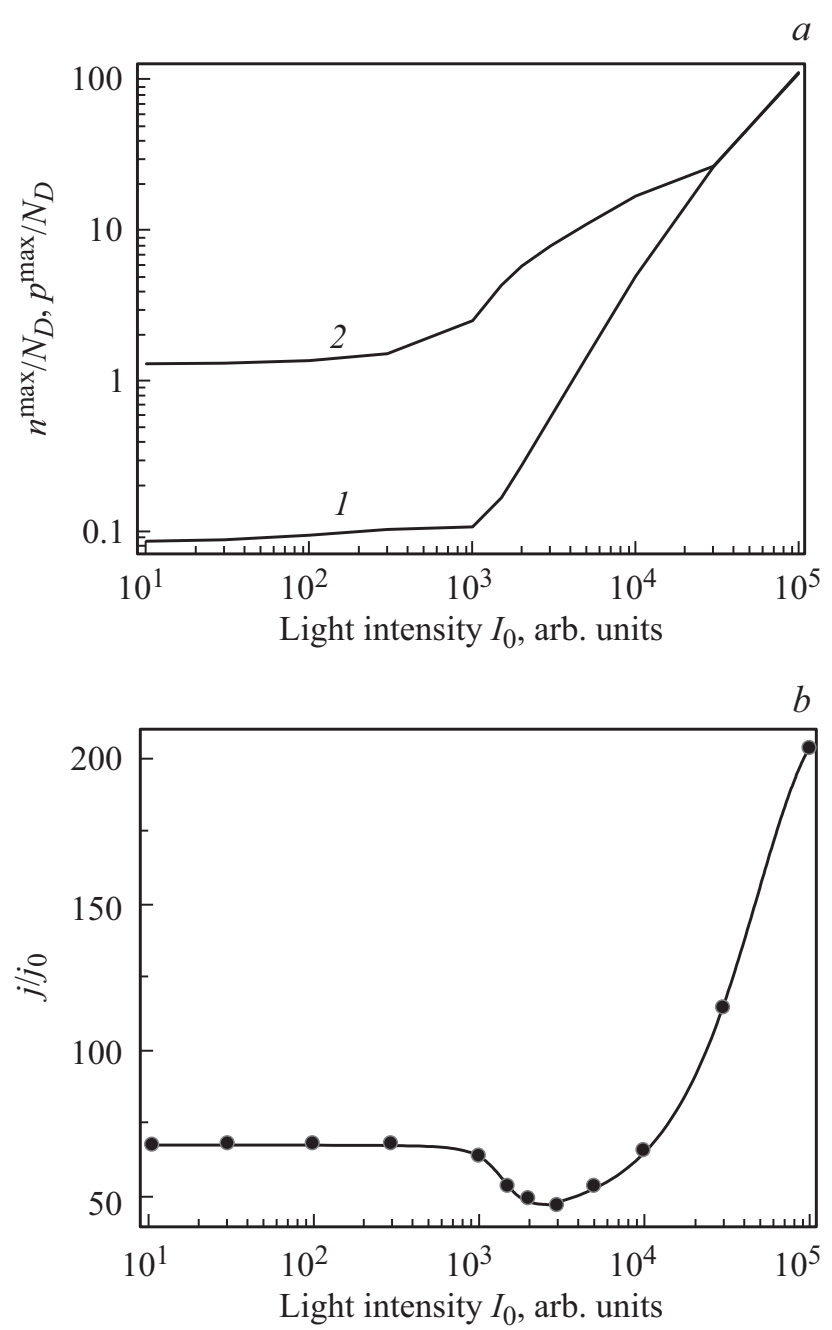

Рис. 5. Максимальные значения концентраций электронов $n^{\max }(1)$ и дырок $p^{\max }(2)$ в потенциальных ямах InAs $(a)$ и величина возбуждаемого в структуре фототока $(b)$ в зависимости от интенсивности света $I_{0}$, падающего на образец с параметрами $N_{D}=1 \cdot 10^{18} \mathrm{~cm}^{-3}, \Delta x_{1}=4 \mathrm{HM}, \Delta x_{2}=8 \mathrm{Hм}$.

дырочного тока в системе начинает зависеть от интенсивности засветки, $j\left(\mathrm{I}_{0}\right)$, по закону, напоминающему вид вольт-амперной характеристики диода (рис. $5, b$ ).

\section{5. Заключение}

Рассмотрены электрополевое поведение резонансных особенностей в фототоке и туннельный механизм эмиссии возбуждаемых светом носителей заряда в гетерокомпозициях $\operatorname{InAs}(\mathrm{QD}) / \mathrm{GaAs}$. Показано, что при низких температурах величина фототока в сильном поперечном электрическом поле определяется исключительно эффектом туннелирования электронов сквозь барьер, формируемый в окрестности интерфейсов квантовых точек. Показана возможность вклада в общую величину туннельного тока резонансной составляющей, связанной с туннелированием электронов сквозь барьер с участием локальных уровней дефектов на гетерогранице. Проведен анализ влияния уровня засветки на форму потенциала в структуре, характер распределения носителей заряда в слоях системы и на величину наблюдаемого фототока.

В заключение авторы благодарят А.П. Горшкова и T.О. Мишенко за оказанную помощь при проведении измерений и расчетов. Измерения выполнены на структурах, изготовленных в НИФТИ ННГУ Б.Н. Звонковым.

Работа выполнялась в рамках федеральной целевой программы „Научные и научно-педагогические кадры инновационной России“ на 2009-2013 годы, шифры заявок 2011-1.2.1-12-000-2013-095 и 2012-1.3.1-12-0002003-031 (№ 8543). Авторы также благодарят Российский фонд фундаментальных исследований (проект № 18-42-5200062) за оказанную поддержку.

\section{Список литературы}

[1] Н.Н. Леденцов, В.М. Устинов, В.А. Щукин, П.С. Копьев, Ж.И. Алферов, Д. Бимберг. ФТП, 32, 385 (1998).

[2] И.А. Дмитриев, Р.А. Сурис. ФТП, 35, 219 (2001).

[3] J. Lu, P.T. Webster, S. Liu, Y.H. Zhang, S.R. Johnson, D.J. Smith. J. Cryst. Growth, 425, 250 (2015).

[4] Е.С. Шаталина, С.А. Блохин, А.М. Надточий, А.С. Паюсов, А.В. Савельев, М.В. Максимов, А.Е. Жуков, Н.Н. Леденцов, А.Р. Ковш, С.С. Михрин, В.М. Устинов. ФТП, 44, 1352 (2010).

[5] P.W. Fry, I.E. Itskevich, S.R. Parnell, J. J. Finley, L.R. Wilson, K.L. Schumacher, D.J. Mowbray, M.S. Skolnick, M. Al-Khafaji, A.G. Cullis, M. Hopkinson, J.C. Clark, G. Hill. Phys. Rev. B, 62, 16784 (2000).

[6] G. Torelly, R. Jakomin, L.D. Pinto, M.P. Pires, J. Ruiz, P.G. Caldas, R. Prioli, H. Xie, F.A. Ponce, P.L. Souza. J. Cryst. Growth, 434, 47 (2016).

[7] A. Hospodková, J. Pangrác, M. Zíková, J. Oswald, J. Vyskočil, P. Komninou, J. Kioseoglou, N. Florini, E. Hulicius. Appl. Surf. Sci., 301, 173 (2014).

[8] Б.Н. Звонков, И.А. Карпович, Н.В. Байдусь, Д.О. Филатов, С.В. Морозов. ФТП, 35, 92 (2001).

[9] P. Waltereit, J.M. Fernandez, S. Kaya, T.J. Thornton. Appl. Phys. Lett., 72, 2262 (1998).

[10] В.А. Погребняк, В.М. Яковенко, И.В. Яковенко. ФТТ, 39, 1875 (1997)

[11] Z.S. Gribnikov, K. Hess, G.A. Kosinovsky. J. Appl. Phys., 77, 1337 (1995).

[12] P.M. Mensz, S. Luryi, A.Y. Cho, D.L. Sivco, F. Ren. Appl. Phys. Lett., 57, 2558 (1990).

[13] М.Л. Орлов, Л.К. Орлов. ФТП, 43, 679 (2009).

[14] T. Sugaya, K.Y. Jang, C. Koo, K. Komori, A. Shinoda, K. Yonei. J. Appl. Phys., 97, 034507 (2005).

[15] L.K. Orlov, A.A. Mel'nikova, M.L. Orlov, N.A. Alyabina, N.L. Ivina, V.N. Neverov, Zs. Horvath. Physica E: Low Dimensional Systems \& Nanostructures, 51, 87 (2013).

[16] C.M.A. Kapteyn, F. Heinrichsdorff, O. Stier, R. Heitz, M. Grundmann, N.D. Zakharov, D. Bimberg, P. Werner. Phys. Rev. B, 60, 14265 (1999)

[17] Н.С. Волкова, А.П. Горшков, Д.О. Филатов, Д.С. Абрамкин. Письма ЖЭТФ, 100, 175 (2014). 
[18] И.А. Карпович, Б.Н. Звонков, С.Б. Левичев, Н.Б. Байдусь, С.В. Тихов, Д.О. Филатов, А.П. Горшков, С.Ю. Ермаков. ФТП, 38, 448 (2004).

[19] Э.Н. Король. ФТТ, 19, 1266 (1977).

[20] Р.А. Сурис. ФТП, 20 (11), 2008 (1986).

Редактор Л.В. Шаронова

Field effect behavior of the resonances in the photocurrent tunneling component

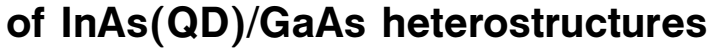

M.L. Orlov ${ }^{1,3}$, N.S. Volkova ${ }^{2}$, N.L. Ivina ${ }^{1}$, L.K. Orlov ${ }^{3,4}$

${ }^{1}$ RANEPA

603950 Nizhny Novgorod, Russia

${ }^{2}$ Lobachevsky State University of Nizhny Novgorod, 603950 Nizhny Novgorod, Russia

${ }^{3}$ Institute for Physics of Microstructures, Russian Academy of Sciences, 603950 Nizhny Novgorod, Russia

${ }^{4}$ Nizhny Novgorod State Technical Alekseev University 603950 Nizhny Novgorod, Russia

Abstract The work focuses on two objectives. First is study of the behavior of electric-resonance features observed in the photovoltaic characteristics of InAs/GaAs heterostructures. Second is identification of the emission mechanism of charge carriers which are excited by light from InAs quantum dots into the GaAs matrix. The photocurrent is defined solely by electron tunneling through the potential barrier at the layers boundary at the presence of a strong electric field. Comparison of the experimental curves with the quasi-classical expression for the tunneling current component and analysis of potential structure have led to the refinement of some parameters of investigated heterostructures and revelation of the resonant component associated with the tunneling of electrons through the barrier with participation of local defect levels at the interface. Theoretical analysis has been carried out to study the effect of the exciting level of the electron subsystem on the photocurrent flowing throw burrier between InAs QD and GaAs. 